\title{
La evolución en una serie temporal de libros de texto (1945-2016): Cambios en la metodología y en la presencia de concepciones alternativas
}

\author{
Alejandro Pastor Pastor ${ }^{1}$, Rubén Limiñana Morcillo² \\ ${ }^{1}$ Centro Público Integrado de Formación Profesional Batoi. Alicante. España. \\ ${ }^{2}$ Departamento de Didáctica General y Didácticas Específicas. Universidad de Alicante, \\ Alicante. España
}

[Recibido el 30 de noviembre de 2018, aceptado en el 13 de septiembre de 2019]

\begin{abstract}
Se analiza el tema de la evolución en una serie de libros de texto desde 1945 hasta 2016 con el fin de evaluar la presencia de concepciones alternativas y los cambios producidos en cómo se presentan los contenidos del tema. Además, se analiza si existe alguna tendencia relacionada con la legislación educativa española. Este análisis ha dado lugar a varios resultados de interés. Entre ellos, se detecta un aumento en el contenido sobre historia del pensamiento evolutivo, número de actividades y número de figuras, lo que podría estar relacionado con cambios en la metodología. Pese a ello, la presencia de concepciones alternativas se mantiene y son pocos los intentos detectados en que se produzca una confrontación con ellas. Por ello no parece que haya posibilidad de que se dé un cambio conceptual significativo, ya que no se cumple la condición necesaria de "insatisfacción con la conceptualización existente".
\end{abstract}

Palabras clave: Cambio conceptual; metodología didáctica; concepciones alternativas; errores conceptuales; didáctica de la biología

\section{The evolution in text books (1945-2016): Changes in methodology and in the presence of misconceptions}

We analyzed the topic of evolution in a set of biology textbooks from 1945 to 2016 . The objectives of this study were to evaluate changes in the presence of misconceptions, as well as to analyze how contents about evolutions are presented. Additionally, we analyze tendencies on these aspects related to Spanish educational legislation. Results showed an increase in the treatment of history on the evolutionary thought, number of activities and number of figures, which might be related to changes in the teaching methodology. However, misconceptions remain in textbooks and there are few attempts to produce confrontation with them. Thus, it seems that the possibility of generating a significant conceptual change is low, since the necessary condition of "dissatisfaction with the current conceptualization" is not met.

Keywords: Conceptual change; educational methodology; misconceptions; conceptual errors; biology education.

Para citar el artículo. Pastor Pastor, A. y Limiñana Morcillo, R. (2019). La evolución en una serie temporal de libros de texto (1945-2016): Cambios en la metodología y en la presencia de concepciones alternativas. Ápice. Revista de Educación Científica, 3(2), 71-92. DOI: https://doi.org/10.17979/ arec.2019.3.2.4811

Contacto: ale.pastor37@gmail.com 


\section{Introducción}

En las últimas décadas se han producido cambios en la legislación educativa española. En 1970 con el final de la dictadura se manifiesta la necesidad de profesionalizar la educación para que el país pueda adaptarse a las circunstancias internacionales, promulgándose la Ley General de Educación para sustituir a la Ley de Instrucción Pública (Ley Moyano, 1857). Otro punto de inflexión clave de nuestro sistema se produce en 1990 con la Ley Orgánica General del Sistema Educativo (LOGSE), momento en el que se hace patente la necesidad de modificar la metodología para adaptarla a las condiciones generadas en la transición (San Román, 2013). Ya en el siglo XXI, se promulga la Ley Orgánica de Educación (LOE), todavía vigente con la modificación posterior mediante la Ley Orgánica para la Mejora de la Calidad Educativa (LOMCE) en 2013.

Estos cambios, pese al enorme componente ideológico que han tenido, también se han centrado en la metodología (San Román, 2013). En este contexto, los libros de texto se han perpetuado como el material principal sobre el que muchos profesores desarrollan su tarea (Perales y Jiménez, 2002), constituyéndose en el canal mediante el que se presentan didácticamente los contenidos del currículo oficial y reflejando los valores y la concepción del mundo en cada etapa (Martínez Bonafé, 2002; Occelli y Valeiras, 2012). Serían, por lo tanto, un reflejo de los cambios en los modelos educativos y en las innovaciones metodológicas.

\section{Objetivos}

Las preguntas de investigación que guían este trabajo son: ¿aparecen concepciones alternativas sobre la evolución en los libros de texto? Si así fuera, ¿̇ha variado la presencia de concepciones alternativas sobre la evolución en las últimas décadas en los libros de texto? ¿Se han producido cambios en la forma de presentar el tema de la evolución en los libros de texto? ¿Están estos cambios relacionados con cambios legislativos? Para tratar de responder a estas preguntas nos planteamos dos objetivos específicos:

Objetivo 1: Cuantificar la presencia de concepciones alternativas asociadas a la evolución en libros de texto de Biología desde el año 1945 hasta el 2016 y evaluar su tendencia.

Objetivo 2: Analizar cómo se presenta la evolución en una secuencia de libros (19452016), discutir si existen cambios en la metodología y si estos cambios tienen relación con el modelo educativo vigente. Para ello se evaluará el contenido dedicado a historia del pensamiento evolutivo, el número y tipo de actividades y la presencia y tipo de figuras.

\section{Marco teórico}

\section{¿Por qué la evolución?}

"Nada tiene sentido en biología si no es a la luz de la evolución" (Dobzhansky, 1973). La teoría de la evolución removió las entrañas de la sociedad y generó una fecunda sucesión de discusiones y novedades científicas y argumentales. Desde las confrontaciones de Cuvier y Lamarck entre fijismo y evolucionismo hasta la Síntesis Expandida, el pensamiento evolutivo ha sido paradigmático en términos de metodología científica y argumental y en la validación y construcción de teorías y modelos que expliquen los fenómenos naturales (Álvarez-Pérez y Ruiz-Gutiérrez, 2015). Por este motivo, la evolución también resulta un buen modelo de aprendizaje (Hernández, Álvarez-Pérez y Ruiz-Gutiérrez, 2009). Pese a ello, se trata de un entramado de conocimiento tan amplio que resulta complejo definir qué aspectos son los más relevantes y cómo enseñarlos (Álvarez-Pérez y Ruiz-Gutiérrez 
2015). El resultado de esto es que los contenidos de los libros de texto se han centrado excesivamente en el detalle, forzando a los estudiantes a optar por la memorización de conceptos segregados y con poca compresión de su integración y, a veces, con importantes concepciones alternativas o errores conceptuales (Butler, Mooney y O'Grady, 2015; Occelli y Valeiras, 2012).

\section{¿Qué son las concepciones alternativas?}

En la década de 1970 se constató que muchos estudiantes no comprendían conceptos científicos básicos, pese a la insistente repetición con que habían sido enseñados (Viennot, 1979; Solbes, 2009). Esta carencia se manifiesta con la presencia de ideas que difieren de los conceptos científicamente aceptados (Maskiewicz y Lineback, 2013). Estas ideas fueron denominadas misconceptions en inglés y traducidas como concepciones alternativas, y han recibido muchas definiciones: "ideas inexactas que emergen de la instrucción", "un entendimiento alternativo erróneo construido en respuesta al conocimiento previo" o, simplemente, como ideas naifs o erróneas (Crowther y Price, 2014; Munson, 1994). Aquí las definiremos como "ideas científicamente inexactas sobre un concepto científico" (Andrews et al., 2012). Las concepciones alternativas son similares para estudiantes de distintos países y resistentes a ser sustituidas por conocimientos científicos en la enseñanza habitual (Pintó, Aliberas i Maymí y Gómez Carrillo, 1996; Balgopal, 2014). Diversos estudios muestran que existe un gran número de concepciones alternativas persistentes sobre evolución, por ejemplo, en selección natural (Bishop y Anderson, 1990; Nehm y Reilly, 2007; Gregory 2009; González-Galli y Meinardi, 2017), deriva genética (Andrews et al., 2012) o macroevolución (Catley y Novick, 2009).

Desde la educación formal, una de las principales fuentes de generación de concepciones alternativas en los alumnos son los libros de texto (van den Broek y Kendeou, 2008) que, en este sentido, tienen un papel paradójico. Por un lado, son el material principal sobre el que se desarrolla el proceso de enseñanza-aprendizaje (Jiménez-Aleixandre, 1994), pero, por otro lado, se ha demostrado que pueden contener errores científicos, explicaciones engañosas, actividades inadecuadas, y problemas en la secuenciación de conceptos que podrían fomentar que los alumnos construyan y mantengan ideas erróneas (Woodward y Elliot, 1987; Linhart, 1997; Stern, 2004; Rees, 2007; King, 2010). Es crítico, por tanto, que autores, editores y profesores estén alerta para localizar la presencia de concepciones alternativas en los libros de texto que interactúen con el aprendizaje, ya sea para eliminarlas o para reconducirlas en el proceso de enseñanza-aprendizaje (Tekkaya, 2002; Van den Broek y Kendeou, 2008).

La investigación didáctica ha arrojado cuantiosos resultados acerca de la presencia de concepciones alternativas y cómo tratarlas en los libros de texto (por ejemplo, Bishop y Anderson, 1990; Gibson, 1996; van den Broek y Kendeou, 2008). Pese a ello, no hemos encontrado registro de estudios que realicen un análisis temporal comparativo entre libros de texto de diferentes años que permita inferir si se está produciendo un cambio en este sentido, lo cual podría tener un efecto importante en el proceso de enseñanza y aprendizaje.

Hasta hace poco, el consenso general era que las concepciones alternativas debían ser erradicadas, ya que generaban problemas en el aprendizaje (Maskiewicz y Lineback, 2013). Más tarde se sugirió que la erradicación de las concepciones alternativas, sin una discusión previa que permita esclarecerlas o sustituirlas por conocimiento contrastado, contradice los principios del constructivismo (Smith, Disessa y Roschelle, 1993), ya que uno de sus principales pilares es el aprendizaje mediante la transformación y reconstrucción del conocimiento previo en ideas más acertadas (Maskiewicz y Lineback, 2013). En 
este contexto, las concepciones alternativas deberían ser "una oportunidad para la disonancia que podría utilizarse para el progreso educativo" (Crowther y Price, 2014). Esto sería, por ejemplo, el modelo de cambio conceptual (Posner, Strike, Hewson y Gertzog, 1982) que estipula que para que se dé una modificación constructiva de los errores debe existir: 1) insatisfacción con los conceptos existentes; 2) disponibilidad de un nuevo concepto inteligible; 3) este nuevo concepto debe ser demostrable; y 4) percibido como útil. Sin embargo, el modelo inicial de cambio conceptual ha recibido críticas ya que no tiene en cuenta aspectos motivacionales y creencias (Linnenbrink y Pintrich, 2002), pese a que estos factores son relevantes en el proceso de aprendizaje (Balgopal, 2014). Así surgió el modelo de reconceptualización del conocimiento que intentaba compatibilizar el cambio conceptual con otras teorías como el aprendizaje situado (Dole y Sinatra, 1998), que pretendía acercar los conocimientos al contexto de los alumnos. Por otra parte, el modelo de enseñanza por indagación, que persigue el desarrollo de conocimientos y comprensión de las ideas científicas a través de unidades problematizadas (NRC, 1996), conforma otra propuesta en línea con el marco del cambio conceptual (Ibáñez y Martínez-Aznar, 2005). Para ello debe cumplirse que 1) los conocimientos tengan un interés científico y creen un conflicto cognitivo; 2) impliquen formulación y comprobación de hipótesis y problemas reales; 3 ) construcción de modelos aplicables; y 4) evaluación conjunta del proceso de enseñanza-aprendizaje (NRC, 1996).

Sin embargo, el calado de estos modelos en el sistema educativo es dudoso. Además, estudios de diversos países reflejan dificultades en el aprendizaje de conceptos relacionados con la evolución (Hiatt et al., 2013; Álvarez-Pérez y Ruiz-Gutiérrez, 2015). Los libros de texto, como reflejo del modelo educativo de cada época, deberían incorporar las innovaciones didácticas que se producen. Sin embargo, son varios los autores que han señalado importantes carencias en los libros, destacando: falta de precisión conceptual y de visión histórica (Jeffery y Roach, 1994; Álvarez-Pérez y Ruiz-Gutiérrez, 2015), persistencia de actividades poco prácticas y creativas (Martínez-Losada y García-Barros, 2003; Pardo, 2004), profusión de figuras confusas, desconectadas del texto e incompletas (Perales y Jiménez 2002) y permanencia de ideas incorrectas (Occelli y Valeiras, 2012). Pese a ello, son pocos los trabajos que efectúan un análisis temporal para discutir si se ha producido y cómo la incorporación de estos modelos didácticos a lo largo de las últimas décadas, y si su inclusión ha mejorado la calidad de los libros. En este sentido, realizaremos un análisis de variables como tipo de actividades y figuras predominante, aparición de contenido histórico-teórico y cantidad de concepciones alternativas en el tema de evolución de una serie de libros de 1945 a 2016, con el fin de comprobar si se han producido cambios en estos aspectos.

\section{Materiales y métodos}

\section{Revisión bibliográfica sobre el pensamiento evolutivo}

Realizamos una revisión bibliográfica abarcando la mayor cantidad de trabajos posible (Gould, 1980; Mayr, 1982; Hey, 1999; Shtulman, 2006; Müller, 2007; Jablonka y Lamb, 2010; Pigliucci y Finkelman, 2014; Futuyma, 2015) para identificar elementos fundamentales en la historia del pensamiento evolutivo (Fig. 1).

\section{Material de estudio}

Recopilamos una muestra de 32 libros de texto, desde el año 1945 hasta el año 2016 (ver Tabla ANEXA 1 en material suplementario con los datos de los libros analizados). Clasificamos los libros en función de la ley educativa con la que se publicaron, intentando abarcar la máxima variedad posible de editoriales y cursos en cada etapa legislativa, para 


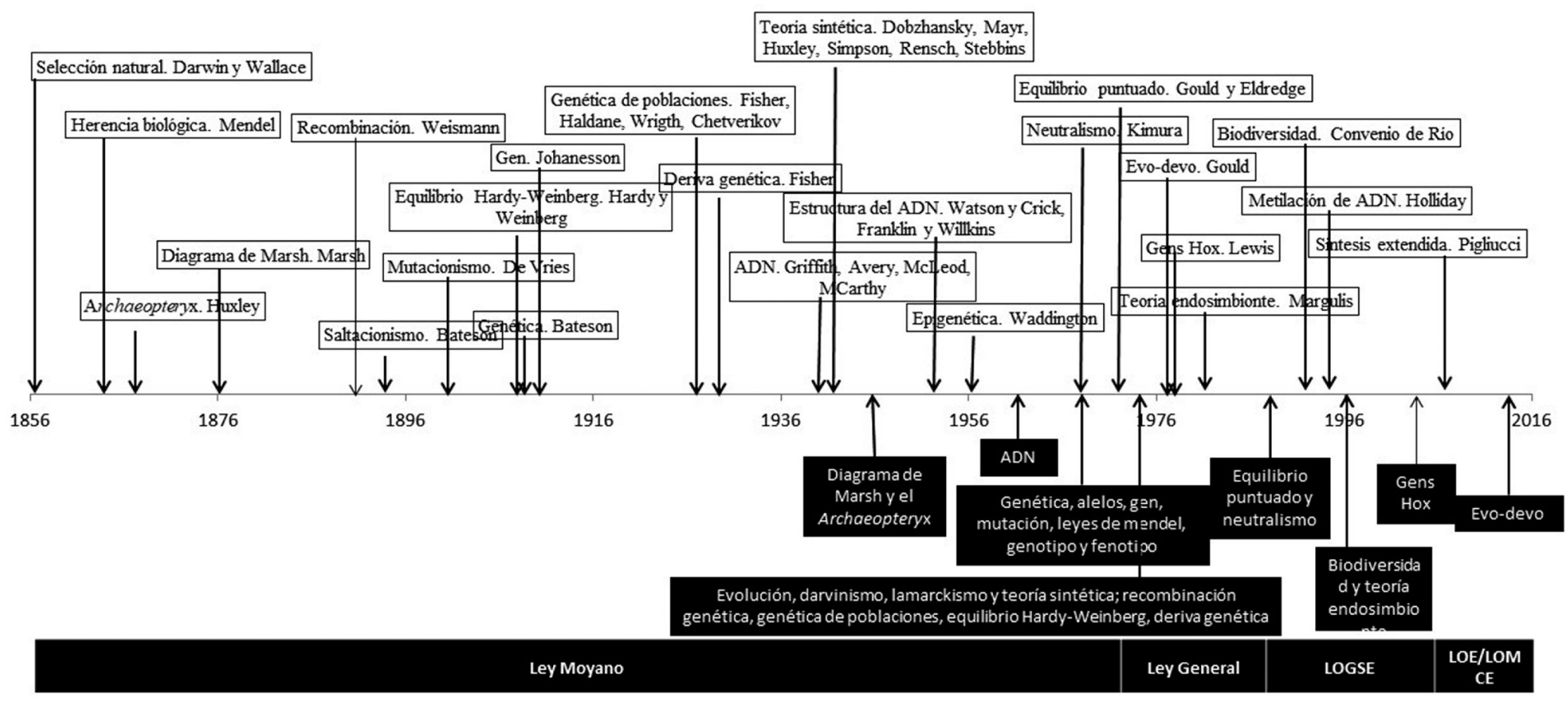

Figura 1. Línea del tiempo en la que se muestra la fecha de publicación de algunos de los principales descubrimientos en evolución (cuadros de color blanco), aparición de la primera cita a estos conceptos en los libros estudiados (recuadros negros), y correspondencia cronológica con las etapas legislativas (cuadros negros)

obtener finalmente un tamaño muestral de ocho libros por etapa. Las etapas legislativas contempladas fueron: 1) Ley Moyano (hasta 1970). 2) Ley General de Educación (19701990). 3) LOGSE (1990-2002). 4) LOE/LOMCE (2006-Actualidad). Esta última etapa pretendía inicialmente ser dos (una LOE y otra LOMCE); sin embargo, pese a que la LOMCE modifica a la LOE, los cambios en el currículo no afectan significativamente a los contenidos de las unidades didácticas de evolución. En la Fig. 1 mostramos la correspondencia cronológica de las diferentes etapas legislativas con los principales descubrimientos en evolución. Algunas de las fechas de publicación de los libros parecen no corresponderse con la etapa legislativa en la que están clasificados; esto se debe a que, pese a que había cambiado la ley, su aplicación no se produjo inmediatamente.

Para cada etapa, los libros de diferentes cursos los clasificamos en libros de mayor nivel formativo (Ley Moyano: 3ㅇ y 5o de BAC; Ley General: COU; LOGSE y LOE/LOMCE: 1을 BAC) y menor nivel formativo (Ley Moyano: 1ㅇ y 2ㅇ de BAC; Ley General: 8ㅇ EGB y 1ㅇ BUP; LOGSE y LOMCE: 4 ESO), intentando que hubiera un tamaño muestral igual para cada nivel dentro de cada etapa legislativa $(n=4)$; pese a ello en la etapa de la Ley General no fue posible y quedó desproporcionada (superior: $n=3$ frente a inferior: $n=5$ ). Consideramos que este tamaño muestral resulta suficiente para evaluar la existencia de diferencias entre niveles formativos dentro de cada etapa. En las últimas tres etapas los niveles se corresponden entre sí; sin embargo, en la etapa Moyano se trata de niveles inferiores debido a que no pudimos contar con una mayor o más variada muestra para esta etapa. No obstante, consideramos que resulta interesante incluir estos libros, ya que no generan ningún cambio significativo en los resultados.

En general, el principal criterio de selección de libros y cursos fue que la evolución se tratara como una unidad didáctica independiente del resto, lo cual ocurre en 40 ESO y 10 
de Bachillerato para las etapas LOE/LOMCE y LOGSE, y en COU y 10 de BUP para la etapa de la Ley General (en esta etapa también se analizó un libro de 8 de EGB, con el fin de abarcar mayor variedad). Sin embargo, en la etapa legislativa correspondiente a la ley Moyano no fue posible, ya que la evolución no aparecía como una unidad didáctica; en estos libros buscamos las páginas en las que se trataban temas relacionados con la evolución (por ejemplo, clasificación de los seres vivos, historia geológica o adaptaciones de los seres vivos al medio) y analizamos estas páginas como si constituyesen una unidad didáctica.

En resumen, en cada etapa legislativa se analizaron ocho libros de diferentes editoriales y cursos con el fin de poder realizar un análisis estadístico comparativo entre etapas. También analizamos la influencia del nivel formativo en las distintas variables analizadas, aunque su influencia resulta limitada respecto a la de la etapa legislativa, por lo que no siempre se muestran los resultados de este último análisis.

\section{Variables medidas}

Concepciones alternativas: realizamos una revisión bibliográfica sobre concepciones alternativas en evolución, basándonos en aquellas que son más habituales en los libros de texto (Tshuma y Sanders, 2014) y en el alumnado (Hiatt et al., 2013). Clasificamos las concepciones alternativas en cinco tipos, siguiendo a Hiatt et al. (2013). En la Tabla 1 se aporta la clasificación y ejemplos hallados en los libros.

Historia del pensamiento evolutivo: en cada libro cuantificamos el número de páginas (y porcentaje del tema) dedicadas a historia, así como el número de teorías y autores citados, en total y por página. Consideramos páginas dedicadas a historia aquellas en las que se trata el pensamiento evolutivo a través de la descripción de teorías, descubrimientos o razonamientos. En el material anexo incluimos dos tablas (tabla ANEXA 2 y tabla ANEXA 3) en las que se muestra qué teoría y autores fueron citados en cada libro analizado.

Figuras: cuantificamos el número de figuras y las clasificamos en dos tipos: 1) figuras con contenido aclarativo, complementario o de ampliación (esquemas, tablas, gráficas...); 2) figuras sin contenido aclarativo, complementario o de ampliación (fotografías, dibujos, viñetas...). Calculamos el número de figuras por página y qué porcentaje de ellas contenían información complementaria. Como ejemplos de figuras con contenido aclarativo hemos considerado, además de gráficas o tablas, aquellas en que se especifican aspectos de la evolución o se aportan pruebas del proceso evolutivo (Tabla ANEXA 4).

Actividades: cuantificamos el número actividades totales y por página, clasificándolas en: 1) iniciales (para promover la formulación de hipótesis, "¿Crees que los organismos vivos han existido siempre tal cual los vemos ahora?"); 2) de memorización (copiar las respuestas directamente del texto, “¿Qué explicación daba Cuvier a la presencia de organismos fósiles que ya no existen?"); 3 ) de razonamiento (resolución de una cuestión no explícita en el texto, "en los últimos años se ha detectado un incremento de insectos parásitos e infecciosos resistentes a los insecticidas, por ello las empresas encargadas de fabricarlos están continuamente investigando otros nuevos. Propón una explicación a este fenómeno"); 4) relacionadas con las tecnologías de la información y la comunicación (TIC; "la historia evolutiva de un depredador, como el león, y su presa, por ejemplo, las cebras, están relacionadas. Busca información y explica qué tipo de fenómeno se produce"); 5) lecturas complementarias; y 6) presentan una concepción alternativa para conseguir un cambio conceptual ("es frecuente oír frases como: 'si continuamos cortándole la cola a los perros bóxer pronto nacerán con una cola más corta'. Intenta buscar otras frases de la vida cotidiana en que se sobreentienda la idea de los caracteres adquiridos"). 
Tabla 1. Ejemplos de concepciones alternativas detectadas y autor que las definió

\begin{tabular}{llc}
\hline Concepción alternativa & Ejemplo en los libros & Autor \\
\hline \multicolumn{1}{c}{ 1. Biológicas comunes } & & \\
\hline $\begin{array}{ll}\text { Atribuir propósito a organismos, } \\
\text { ambiente... }\end{array}$ & "Los organismos utilizan el mimetismo & Hiatt et al., \\
& $\begin{array}{l}\text { para adoptar el aspecto de otro } \\
\text { organismo " }\end{array}$ & 2013 \\
Carácter negativo a organismos, ambiente... "El ambiente es adverso" & "
\end{tabular}

\section{Teoría sintética}

Los caracteres no usados se pierden "Degeneraban los demás dedos"

Hiatt et al., 2013

Herencia de caracteres adquiridos

"Caracteres adquiridos por mutación se heredan"

Ignorar cambios neutros no adaptativos

"Las mutaciones son malas la mayoría de las veces, pero pueden ser buenas"

Ignorar la posibilidad de la deriva genética "Sólo la selección natural dará lugar a nuevas especies"

La selección natural no actúa sobre "La selección no cesa de elegir a los fenotipo que tienen una combinación génica beneficiosa"

Los organismos más antiguos son más complejos, o la inversa

"Ley de diversidad progresiva de los seres"

Selección natural como un carácter que incrementa el éxito, una mutación que "La selección es la lucha por la existencia"

mejora especies, la supervivencia del más adaptado, competencia o el ambiente eligiendo...

La evolución es consecuencia del ambiente "El estrés produce adaptaciones"

Tshuma y Sanders, 2014

Los organismos se adaptan durante su vida "Las adaptaciones son cambios de los para sobrevivir organismos para adecuarse a la vida"

Los individuos inician y controlan el cambio

"Las plantas reducen sus hojas"

"I

$"$

La población tendrá el carácter favorable

"Finalmente todas tienen cuellos largos"

La evolución es a mejor o más complejo

"La complicación de las especies se va consiguiendo por evolución"

La evolución es lineal

Diagrama de Marsh

\section{Este estudio}

El aislamiento produce variabilidad genética

"El aislamiento reproductor implica cambios genéticos"

Este estudio

\section{Evo-devo}

Referencias erróneas al desarrollo

"Los intentos para demostrar la herencia de los caracteres adquiridos

Hiatt et al., 2013

El fenotipo es sólo el producto de los han fracasado" genes

"Sólo genotipos diferentes dan lugar a individuos con distintas características"

Los cambios fenotípicos solo ocurren "Sólo nuevos genes generan cuando los genes aparecen o desaparecen variabilidad" 
Tabla 1. Ejemplos de concepciones alternativas detectadas y autor que las definió. (Continuación)

\begin{tabular}{llc}
\hline \multicolumn{1}{c}{ 4. Explicaciones confusas } & & \\
\hline Uso incorrecto de "adaptación" & $\begin{array}{l}\text { "Las respuestas adaptativas no se } \\
\text { registran en los genes" }\end{array}$ & $\begin{array}{c}\text { Tshuma y } \\
\text { Sanders, } 2014\end{array}$ \\
Uso incorrecto de "evolución" & $\begin{array}{l}\text { "Es el desarrollo de caracteres para } \\
\text { resolver mejor los problemas" }\end{array}$ & " \\
Uso incorrecto de "especiación" & $\begin{array}{l}\text { "Diferencias geográficas en la } \\
\text { mutación" }\end{array}$ & Este estudio \\
Otros errores conceptuales & $\begin{array}{l}\text { "Ancestro común reunía las } \\
\text { características de las especies que } \\
\text { descienden de él" }\end{array}$ & Este estudio \\
& &
\end{tabular}

Dios como explicación "El hombre creado a imagen de Dios..." Este estudio

\section{Análisis estadístico}

El objetivo de este análisis es comparar entre etapas legislativas e inferir tendencias en la forma en que se presenta el tema de la evolución en los libros de texto. Para ello ponemos a prueba la hipótesis nula $\left(\mathrm{H}_{0}\right)$ de que no existen diferencias entre las etapas legislativas para las variables medidas. Con el fin de rechazar o no esta hipótesis, aplicamos diferentes análisis de comparación de medias dependiendo de si los datos cumplen los supuestos de homocedasticidad y normalidad. Para comprobar estos supuestos recurrimos a la prueba de Levene (homocedasticidad) y la de Shapiro-Wilks (normalidad) a un nivel de significación de 0,05. Aplicamos ANOVA de dos factores (etapa legislativa y nivel formativo) para comparar entre etapas en las variables que cumplen estos supuestos, bien para las variables transformadas o sin transformar. Estas variables fueron: número de concepciones alternativas por página (sin transformar), número total de concepciones alternativas sobre evolución (transformación logarítmica), variables relacionadas con la historia del pensamiento evolutivo (páginas dedicadas a la historia y su porcentaje respecto al tema de la evolución, número de citas a autores, citas por página y número de teorías citadas y teorías por página, que fueron transformadas mediante raíz cuadrada) y número de actividades y actividades por página (sin transformar); en estos casos, la comparación entre medias se realizó mediante la prueba Tukey. Para las variables que no cumplían el supuesto de normalidad, utilizamos la prueba estadística no paramétrica de KruskalWallis; esto se hizo en la comparación entre actividades y tipos de actividades y figuras y tipos de figuras entre etapas legislativas. Para la comparación de medias se obtiene el nivel de significación y las medias \pm error estándar.

\section{Resultados}

\section{Concepciones alternativas}

La Figura 2A muestra un leve aumento de la presencia de concepciones alternativas a lo largo de la serie temporal de libros de texto analizados. Esta tendencia se diluye al comparar las últimas tres etapas legislativas (Fig. 2C), entre las que no existen diferencias ni en el número ni en el tipo de concepciones alternativas. En contraste, el número de concepciones alternativas por página tiende a descender entre la etapa Ley Moyano (anterior a 1970) y la etapa Ley General (1970-1990), donde se registra un punto de inflexión a partir del cual la tendencia se invierte y vuelve a aumentar entre la etapa LOGSE (1990-2006) y la etapa LOE/LOMCE (2006-actualidad) (Fig. 2B y Fig. 2D). Los libros de los cursos inferiores 
muestran mayor cantidad de concepciones alternativas en total que los de mayor nivel formativo $(15,53 \pm 2,01$ y $9,67 \pm 1,04$, respectivamente, $P<0,01)$.
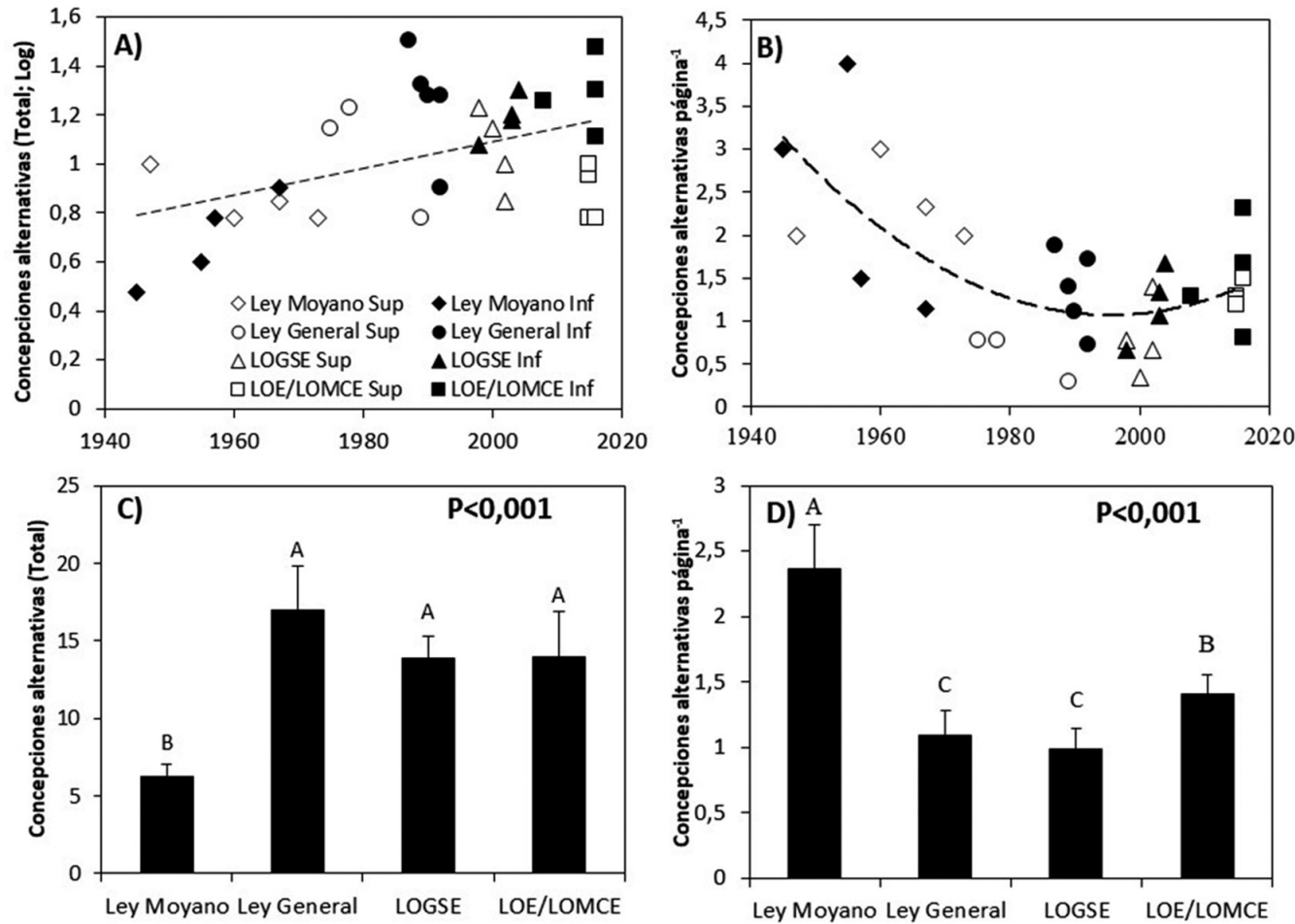

Figura 2. A) Evolución del número total de concepciones alternativas sobre evolución en libros de texto de biología a lo largo de una serie temporal. B) Número de concepciones alternativas sobre evolución encontradas por página a lo largo del tiempo. En A) y B) se muestran los puntos para cada etapa legislativa y nivel (Men.= Menor nivel formativo, May.= Mayor nivel formativo). Las líneas muestran la tendencia. C) Número total de concepciones alternativas en diferentes etapas legislativas (media+ $E E, N=8$ ). D) Concepciones alternativas por página en diferentes etapas legislativas (media+ $E E, N=8$ ). En C) y D), mostramos el nivel de significación del análisis ANOVA ( $\mathrm{P}=\mathrm{P}$-valor) y la comparación de medias mediante el test de Tukey en la que las letras diferentes muestran diferencias entre etapas $(P<0,05)$

En las últimas tres etapas legislativas estudiadas, el tipo de error más común es el relacionado con la teoría sintética (Tabla 2), sin que existan diferencias entre ellas. En la etapa de la Ley Moyano, sin embargo, la principal concepción alternativa se debía a que cualquier fenómeno natural era explicado por obra y gracia de Dios, lo que clasificamos como "otras" concepciones alternativas.

Tabla 2. Concepciones alternativas por libro en las distintas etapas legislativas. Letras diferentes indican diferencias significativas. Se muestra la significación asociada al ANOVA. Los valores son media $\pm \mathrm{EE}(\mathrm{N}=8)$

\begin{tabular}{llllll} 
& $\begin{array}{l}\text { Ley Moyano } \\
\text { (hasta 1970) }\end{array}$ & $\begin{array}{l}\text { Ley General } \\
(\mathbf{1 9 7 0 - 1 9 9 0 )}\end{array}$ & $\begin{array}{l}\text { LOGSE } \\
(\mathbf{1 9 9 0 - 2 0 0 6 )}\end{array}$ & $\begin{array}{l}\text { LOE/LOMCE } \\
\text { (a partir de 2006) }\end{array}$ \\
\hline Biológicas comunes & $0.125 \pm 0.125$ & $1.00 \pm 0.38$ & $0.75 \pm 0.25$ & $1.63 \pm 0.68$ & $\mathrm{~ns}$ \\
Teoría sintética & $1.50 \pm 0.73 \mathrm{~b}$ & $10.50 \pm 1.67 \mathrm{a}$ & $10.63 \pm 1.21 \mathrm{a}$ & $8.13 \pm 2.22 \mathrm{a}$ & $\mathrm{P}<0,001$ \\
Evo-devo & $0.125 \pm 0.125 \mathrm{~b}$ & $3.00 \pm 0.78 \mathrm{a}$ & $1.00 \pm 0.38 \mathrm{~b}$ & $1.13 \pm 0.35 \mathrm{~b}$ & $\mathrm{P}<0,01$ \\
Explicaciones confusas & $1.25 \pm 0.73$ & $2.50 \pm 1.05$ & $1.50 \pm 0.33$ & $2.88 \pm 0.93$ & $\mathrm{~ns}$ \\
Otras & $3.25 \mathrm{a}$ & $0.00 \pm 0.00 \mathrm{~b}$ & $0.00 \pm 0.00 \mathrm{~b}$ & $0.00 \pm 0.00 \mathrm{~b}$ & $\mathrm{P}<0,001$ \\
\hline
\end{tabular}




\section{Historia del pensamiento evolutivo}

En la Tabla 3 mostramos que durante la etapa legislativa de la Ley Moyano (anterior a 1970) no se observa ninguna referencia a la historia del pensamiento sobre la evolución, aspecto que comienza a abordarse en las etapas siguientes. El número de páginas dedicado a la historia y el número de citas a autores aumenta desde la etapa de la Ley General (1970-1990) hasta la etapa LOGSE (1990-2006) donde alcanza un valor máximo significativamente diferente al de las etapas posterior y la anterior (Tabla 3 ).

Para el porcentaje de páginas de historia, el número de citas por página y las teorías evolutivas tratadas la tendencia es semejante, con un pico máximo en la etapa LOGSE (19902006) pero sin diferencias significativas respecto a la etapa anterior y la posterior (Tabla 3). La excepción al comportamiento general lo conforma el número de teorías citadas por página que resulta mayor en la etapa LOE/LOMCE (Tabla 3). Esto puede deberse a que en los libros de mayor nivel formativo de la etapa LOE/LOMCE la historia del pensamiento en evolución se da en información muy concentrada en pocas páginas respecto a los libros de menor nivel formativo de esa misma etapa $(1,83 \pm 1,15$ y $6,00 \pm 0,41$ páginas dedicadas a historia respectivamente, $\mathrm{P}<0,05)$ sin que se registren diferencias significativas en el número total de teorías citadas.

Tabla 3. Diferencias entre etapas legislativas en el número de páginas dedicadas a la historia, el porcentaje que ocupan (\% Historia), el número de citas (Citas), el número de citas por página (Citas/ página), el número de teorías mencionadas (Teorías) y el número de teorías por página (Teorías/ página). Letras diferentes indican diferencias significativas. Se muestra la significación asociada al ANOVA. Los valores son media $\pm \mathrm{EE}(\mathrm{N}=8)$

\begin{tabular}{llllll} 
& $\begin{array}{l}\text { Moyano } \\
\text { (hasta 1970) }\end{array}$ & $\begin{array}{l}\text { General } \\
(\mathbf{1 9 7 0}-\mathbf{1 9 9 0})\end{array}$ & $\begin{array}{l}\text { LOGSE } \\
\mathbf{( 1 9 9 0 - 2 0 0 6 )}\end{array}$ & $\begin{array}{l}\text { LOE/LOMCE } \\
\text { (a partir de 2006) }\end{array}$ \\
\hline Pág. dedicadas historia & $0,0 \pm 0,0 \mathrm{c}$ & $3,5 \pm 0,8 \mathrm{~b}$ & $5,6 \pm 1,3 \mathrm{a}$ & $3,9 \pm 1,0 \mathrm{~b}$ & $\mathrm{P}<0,001$ \\
\% Historia & $0,0 \pm 0,0 \mathrm{c}$ & $17,1 \pm 3,8 \mathrm{a}$ & $19,8 \pm 2,5 \mathrm{a}$ & $17,8 \pm 5,1 \mathrm{a}$ & $\mathrm{P}<0,001$ \\
Citas & $0,0 \pm 0,0 \mathrm{c}$ & $8,4 \pm 2,1 \mathrm{~b}$ & $16,6 \pm 3,3 \mathrm{a}$ & $8,1 \pm 1,8 \mathrm{~b}$ & $\mathrm{P}<0,001$ \\
Citas/página & $0,0 \pm 0,0 \mathrm{~b}$ & $0,5 \pm 0,1 \mathrm{a}$ & $0,8 \pm 0,2 \mathrm{a}$ & $0,8 \pm 0,1 \mathrm{a}$ & $\mathrm{P}<0,001$ \\
Teorías & $0,0 \pm 0,0 \mathrm{~b}$ & $4,6 \pm 0,3 \mathrm{a}$ & $6,4 \pm 0,9 \mathrm{a}$ & $5,4 \pm 0,7 \mathrm{a}$ & $\mathrm{P}<0,001$ \\
Teorías/página & $0,0 \pm 0,0 \mathrm{c}$ & $0,3 \pm 0,0 \mathrm{~b}$ & $0,4 \pm 0,0 \mathrm{~b}$ & $0,6 \pm 0,1 \mathrm{a}$ & $\mathrm{P}<0,001$ \\
\hline
\end{tabular}

La Figura 3 refleja que el número de figuras en los libros de texto sigue una tendencia ascendente (Fig. 3A), con un máximo en la etapa LOE/LOMCE (Fig. 3C).

La misma tendencia se observa en el número de figuras por página (Fig. 3D). El porcentaje de figuras sin contenido tiende a disminuir desde la etapa Ley Moyano a la etapa Ley General, mientras que aumenta desde esta última hasta la etapa LOE/LOMCE (Fig. 3B), por lo que hay un punto de inflexión en la etapa Ley General (así parece indicarlo la elevada variabilidad presente en esa etapa, Fig. 3B). El número de figuras es, en general al, mayor en los periodos LOE/LOMCE y LOGSE (Fig. 3E y F), con el periodo LOGSE mostrando la mayor parte de figuras con contenido aclaratorio, complementario o de ampliación. En este apartado no se obtienen diferencias significativas entre niveles formativos.

\section{Actividades}

El número de actividades en los libros de texto presenta un aumento temporal exponencial (Fig. 4A), con un valor máximo en la etapa LOE/LOMCE y siendo casi inexistentes en la etapa Ley Moyano (Fig. 4C). Como ocurría en el apartado anterior, esta tendencia es 
independiente del nivel formativo. La misma tendencia se observa en el número de actividades por página (Fig. 4D).

El tipo de actividad predominante es de memorización en todas las etapas, siendo en torno al $70 \%$ de las actividades (Fig. 4B). El número máximo absoluto de actividades de memorización por libro se alcanza en las etapas LOE/LOMCE y LOGSE (Fig. 5B). Las actividades de razonamiento encuentran su máximo desarrollo en la etapa LOE/LOMCE (Fig. 5A).
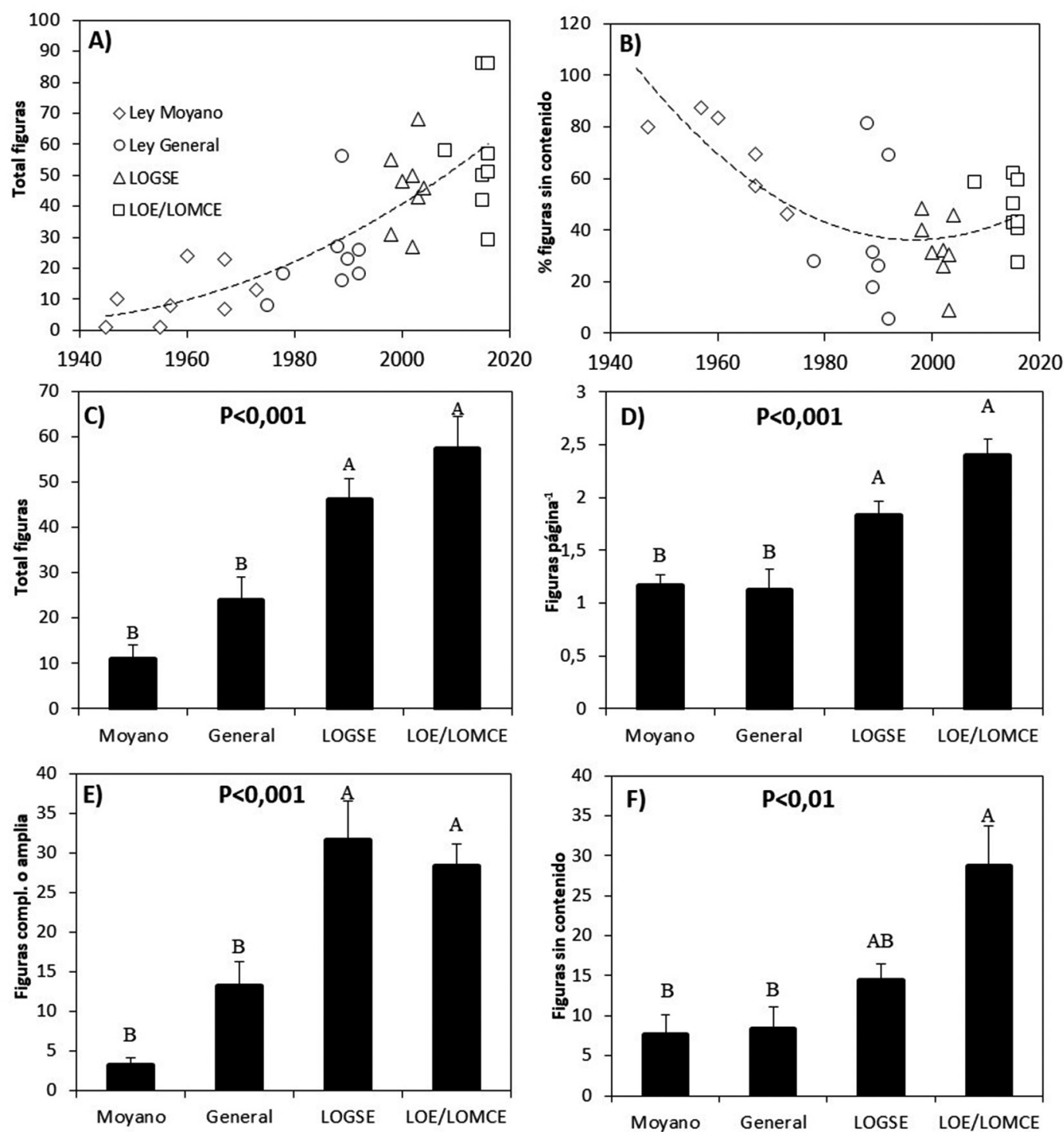

Figura 3. A) Número de figuras totales en los temas de evolución de libros de texto de biología en la serie temporal. B) Evolución del porcentaje de figuras sin contenido explicativo ni complementario a lo largo de la serie. En A) y B) se muestran los puntos para cada etapa legislativa. Las líneas muestran la tendencia. C) Número total de figuras en diferentes etapas legislativas (media+ $\mathrm{EE}, \mathrm{N}=8$ ). $\mathrm{D}$ ) Número de figuras por página en cada etapa (media+ $\mathrm{EE}, \mathrm{N}=$ 8). E) Número de figuras con contenido aclarativo, complementario o de ampliación en cada etapa (media+ EE, $\mathrm{N}=8$ ). $\mathrm{F}$ ) Número de figuras sin contenido aclarativo, complementario o de ampliación en cada etapa (media+ EE, N=8). En C), D), E) y F) mostramos el nivel de significación del análisis no paramétrico Kruskal-Wallis (donde el p-valor del test Shapiro-Wilk fue menor de 0,05 , concluyéndose que no venían de una distribución normal) ( $P=P$-valor) y la comparación en la que las letras diferentes muestran diferencias entre etapas $(P<0,05)$ 
Las actividades relacionadas con las TIC (Tecnologías de la Información y la Comunicación), aparecen en la LOGSE y se confirman en la LOE/LOMCE (Fig. 5C). La presencia de actividades de lectura no ha cambiado en los últimos tres periodos legislativos, pero sí respecto al período Ley Moyano, en el que no aparecían (Fig. 5D). Las actividades relacionas con generar hipótesis iniciales aparecen durante la etapa LOGSE y muestran un máximo en la LOE/LOMCE (Fig. 5E). Las actividades relacionadas con la confrontación de concepciones alternativas aparecen durante la Ley General y muestran un máximo durante la LOGSE para perder presencia en la LOE/LOMCE (Fig. 5F).

\section{Discusión}

\section{Tendencia de las concepciones alternativas}

El número de concepciones alternativas se mantiene estable en las tres últimas etapas educativas, indicando que no se ha producido un intento específico por reducirlas. El aumento del número de concepciones alternativas por página en la última etapa legislativa (LOE/LOMCE) podría ser debido a una excesiva simplificación de la información, ya que, en general, en esta etapa, los libros que presentan mayor número de concepciones alternativas coinciden con que son los que menor cantidad de texto y mayor de figuras presentan. Quizá este esfuerzo por acercar el contenido de la evolución a los alumnos, evitando que los libros sean demasiado densos y recargados de texto (incremento de
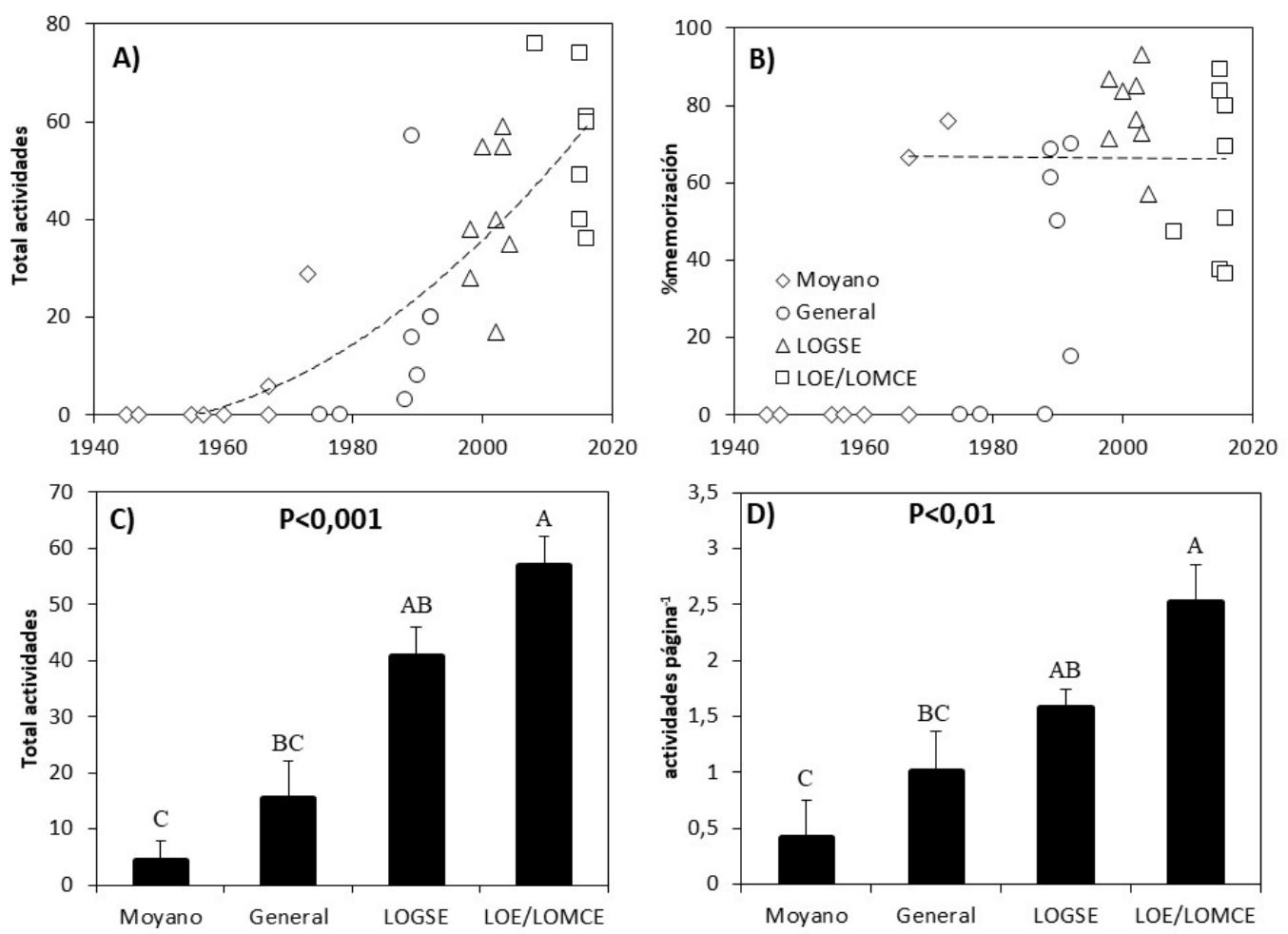

Figura 4. A) Evolución del número de actividades en temas de evolución en libros de biología de la serie temporal. B) Porcentaje de actividades de memorización respecto al total de actividades. En A) y B) se muestran los puntos para cada etapa legislativa. Las líneas muestran la tendencia. C) Actividades totales por libro en temas de evolución en cada etapa. D) Número de actividades por página en cada etapa. (media+ $\mathrm{EE}, \mathrm{N}=8$ ). En $\mathrm{C}$ ) y $\mathrm{D}$ ), mostramos el nivel de significación del análisis ANOVA ( $\mathrm{P}=\mathrm{P}$-valor) y la comparación de medias mediante el test de Tukey en la que las letras diferentes muestran diferencias entre etapas $(P<0,05)$ 
figuras sin contenido, de actividades de respuesta directa, menor cantidad de citas, etc.), implique un menor rigor en los textos en el uso de conceptos en comparación con etapas anteriores.

Exceptuando las concepciones alternativas presentes en la etapa de la ley Moyano (Dios como explicación de la diversidad), los tipos de concepción alternativa se mantienen estables y se muestran como si de un conocimiento científico constatado se trataran, lo que
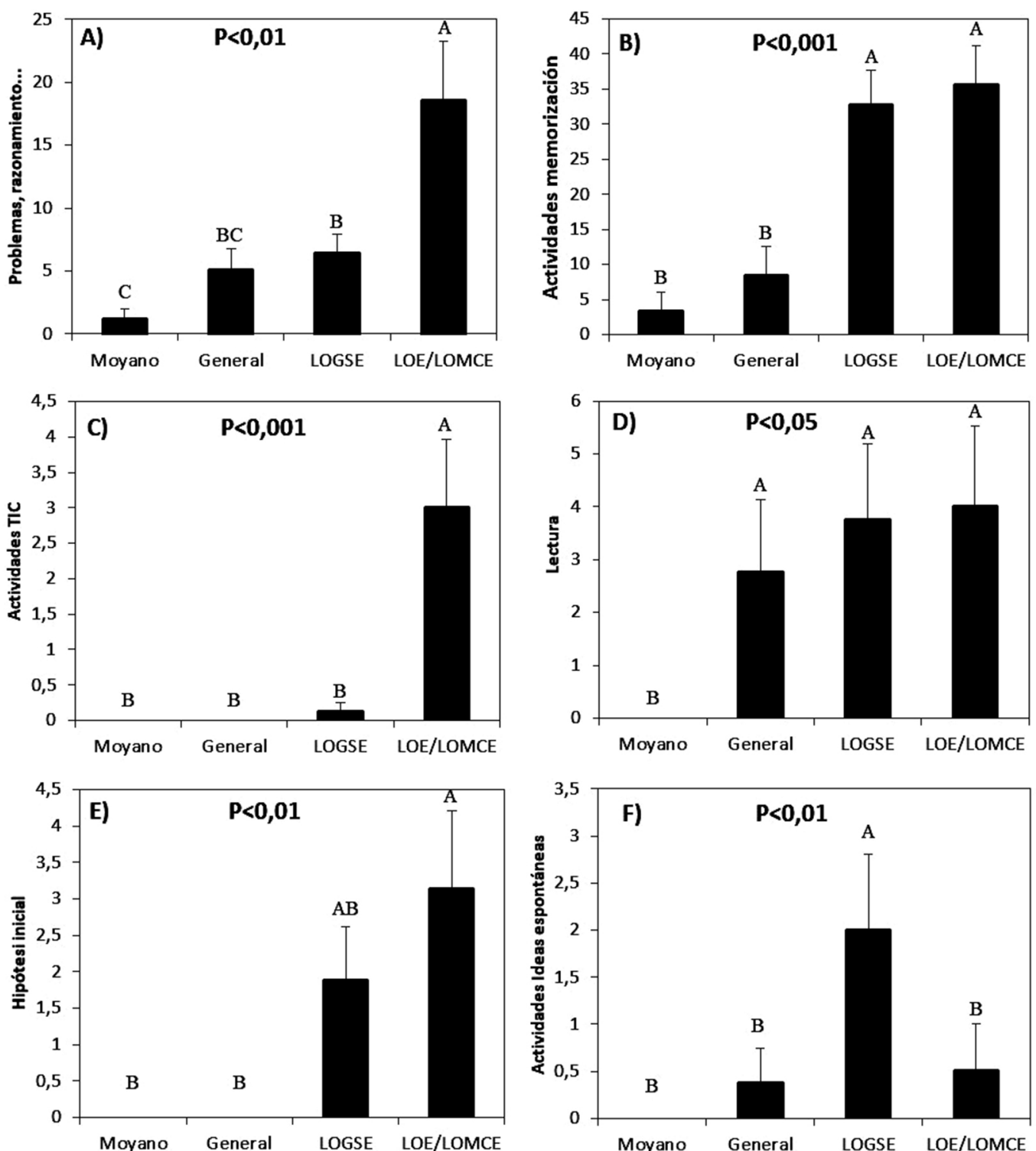

Figura 5. Tipos de actividad en cada etapa legislativa por libro. A) Actividades de razonamiento, problemas, casos prácticos, etc. B) Actividades de memorización (repetición de conceptos o copiar del libro). C) Actividades relacionadas con las TIC (búsqueda en internet, Webquest, etc.). D) Actividades de lectura de textos y comprensión o comentario de texto. E) Actividades de formulación de hipótesis previas a empezar el tema. F) Actividades de confrontación con concepciones alternativas (por ejemplo, análisis de los errores relacionados con el uso y desuso de órganos). Mostramos el nivel de significación del análisis no paramétrico Kruskal-Wallis (donde el p-valor del test Shapiro-Wilk fue menor de 0,05, concluyéndose que no venían de una distribución normal) ( $\mathrm{P}=\mathrm{P}$-valor) y la comparación de medias (media+ $\mathrm{EE}, \mathrm{N}=8$ ) en la que las letras diferentes muestran diferencias entre etapas $(P<0,05)$. 
dificulta su localización y podría ser causa de su persistencia. Además, rara vez se presentan actividades que logren generar una discusión filosófica o argumental respecto a los errores conceptuales más comunes, sino que generalmente se trata de actividades de memorización o repetición que podrían facilitar su presencia (ver apartados siguientes).

\section{Aparición de la historia del pensamiento evolutivo}

La aparición de contenido dedicado a la historia del pensamiento evolutivo en los libros de texto podría ser un intento por clarificar concepciones alternativas resistentes, ya que se ha señalado que la carencia de una visión histórica puede generar confusión (Jeffery y Roach, 1994; Álvarez-Pérez y Ruiz-Gutiérrez, 2015). Hubo un máximo tratamiento de la historia del pensamiento evolutivo durante la etapa LOGSE. En la etapa actual (LOE/ LOMCE) se ha reducido la presencia de la historia en los cursos superiores mientras que en los inferiores se han mantenido niveles parecidos a la etapa anterior. Poner toda esta información al alcance del alumno puede generar un aporte sustancial, ya que las discusiones científicas en torno a la evolución han sido amplias y fecundas. Sin embargo, sería conveniente que las discusiones históricas fueran más completas y estuvieran interrelacionadas, permitiendo que el alumno llegara mediante actividades a las conclusiones que históricamente se han ido alcanzando. En los textos actuales no se llega a producir una confrontación de ideas explícita, y un ejemplo de ello es que un porcentaje elevado de los libros nombra el equilibrio puntuado mientras que a la vez se afirma que la evolución es siempre gradual. ¿Qué utilidad tiene nombrar el equilibrio puntuado como un mecanismo de evolución si no se discute su robustez científica o conveniencia filosófica respecto al gradualismo?

\section{Aumento del número de figuras}

El número de figuras alcanza un valor máximo en la última etapa (LOE/LOMCE). Este aumento podría ser un intento por acercar el contenido al contexto del estudiante con el fin de situar el aprendizaje en un entorno emocional o afectivo más próximo. Algunos autores ya han señalado esta tendencia hacia "el embellecimiento de los libros", indicando que podría generar "dificultades a los lectores" debido a "la proliferación de elementos distractores" que aumentan "el riesgo de interpretaciones erróneas" (Perales y Jiménez, 2002). Una parte significativa de las concepciones alternativas halladas en este trabajo se han encontrado en las figuras y esquemas de los libros más modernos, que son, a la postre, los que menor cantidad de texto y mayor cantidad de figuras poseen.

\section{Cambios en el número y tipo de actividades}

La tendencia a aumentar el número de actividades podría ser una manera de problematizar las unidades didácticas (sensu enseñanza por indagación); sin embargo, las actividades están limitadas a un perfil memorístico y son pocas las que permiten la confrontación de concepciones alternativas. Este problema ya había sido señalado anteriormente, específicamente la casi nula presencia de actividades prácticas pese a su demostrada eficacia (Martínez-Losada y García-Barros, 2003; Pardo, 2004). En la etapa LOE/LOMCE prácticamente no se encuentran actividades de confrontación de concepciones alternativas, aunque sí aumenta el número de actividades de razonamiento y problemas, y las relacionadas con las TIC, mostrando quizá un cambio en la tendencia del tipo de actividad, especialmente hacia situarlas en un contexto que pueda ser más cercano al alumnado y que implique alguna relación con las prácticas científicas. Únicamente en la etapa LOGSE se encontró un número significativo de actividades de confrontación de concepciones alternativas, lo que quizá esté relacionado con la cercanía cronológica a las primeras manifestaciones de la necesidad de cambiar la metodología hacia el "cambio conceptual" (Posner et al., 1982). 


\section{Conclusiones e implicaciones didácticas}

En general, parece que sí existe una relación entre el modelo educativo en boga en el momento de la publicación de los libros y sus características didácticas. En los libros de la etapa LOGSE, por ejemplo, existe una cierta tendencia a que se produzca confrontación de ideas (aumento de actividades relacionadas con concepciones alternativas) que podría ser un intento por generar cambios conceptuales en el alumno. En los libros de la etapa LOE/LOMCE, parece que se produce un intento por problematizar las unidades didácticas, aumentando el número y la variedad de actividades, y de acercar el contenido a los estudiantes aumentando la presencia de fotos, dibujos y viñetas (quizá con el fin de contextualizar el aprendizaje en un entorno más cercano al alumno, sensu reconceptualización del conocimiento de Dole y Sinatra, 1998). Sin embargo, estos cambios no parecen llegar a la raíz del problema y se quedan más en un cambio estético, ya que se sigue manteniendo un modelo didáctico basado en la memorización (elevada presencia de actividades memorísticas o aparición de la historia del pensamiento evolutivo como algo desvinculado de los principales mecanismos relacionados con el proceso evolutivo). Además, la presencia de concepciones alternativas se mantiene y son pocos los intentos detectados por que se produzca una confrontación con ellas. Por ello, no parece que haya posibilidad de que se produzca un cambio conceptual, ya que no se cumple la condición necesaria de "insatisfacción con la conceptualización existente" (Posner et al., 1982).

Como han señalado varios autores (por ejemplo, San Román, 2013; Hiatt et al., 2013; Balgopal, 2014; Álvarez-Pérez y Ruiz-Gutiérrez, 2015) parece necesario seguir profundizando en la adecuación de las herramientas didácticas a las necesidades de los estudiantes de hoy. En el tema que nos ocupa, se podrían diseñar unidades didácticas problematizadas en la que constantemente se exponga al alumnado a una discusión argumentada sobre las concepciones alternativas remanentes en el tema, abordadas desde las prácticas científicas. Para ello, la historia del pensamiento evolutivo es un magnífico sustrato para el uso de un formato didáctico por indagación (por ejemplo, la línea de trabajo propuesta por Acevedo y García-Carmona, 2016), ya que está llena de discusiones científicas de gran calidad argumental, contraposiciones de ideas y razonamientos brillantes, ideas contrastadas y contrastables, y expansiones y revisiones que se han ido sucediendo y siguen en plena expansión.

\section{Referencias}

Acevedo, J. A. y García-Carmona, A (2016). Rosalind Franklin y la Estructura Molecular del ADN: Un caso de historia de la ciencia para aprender sobre la naturaleza de la ciencia. Revista Científica, 25(2), 162-175.

Álvarez-Pérez, E. y Ruiz-Gutiérrez, R. (2016). Proposal for Teaching Evolutionary Biology: A bridge between research and educational practice. Journal of Biological Education, 50(2), 123-146. DOI: https://dx.doi.org/10.1080/00219266.2015.1007887

Andrews, T. M., Price, R.M., Mead, L. S., McElhinny, T. L., Thanukos, A., Perez, K. E., Herreid, C. F., Terry, D. R. y Lemons, P. P. (2012). Biology undergraduates' misconceptions about genetic drift. CBE-Life Sciences Education, 11(3), 248-259. DOI: https://dx.doi. org/10.1187/cbe.11-12-0107

Balgopal, M. M. (2014). Learning and intending to teach evolution: concerns of pre-service biology teachers. Research in Science Education, 44(1), 27-52. DOI: https://dx.doi. org/10.1007/s11165-013-9371-0 
Bishop, B. A. y Anderson, C. W. (1990). Student conceptions of natural selection and its role in evolution. Journal of Research in Science Teaching, 27(5), 415-427. DOI: https://dx.doi.org/10.1002/tea.3660270503

Butler, J., Mooney, G. y O'Grady, A. (2015). An investigation into the prevalence of ecological misconceptions in upper secondary students and implications for preservice teacher education. European Journal of Teacher Education, 38(3), 300-319. DOI: https://dx.doi.org/10.1080/02619768.2014.943394

Catley, K. M. y Novick, L. R. (2009). Digging deep: Exploring college students' knowledge of macroevolutionary time. Journal of Research in Science Teaching, 46(3), 311-332. DOI: https://dx.doi.org/10.1002/tea.20273

Crowther, G. J. y Price R. M. (2014). Re: Misconceptions are "so yesterday!". CBE-Life Sciences Education, 13(1), 3-5. DOI: https://dx.doi.org/10.1187/cbe.13-11-0226

Dole, J. A. y Sinatra G. M. (1998). Reconceptualizing change in the cognitive construction of knowledge. Educational psychologist, 33(2-3), 109-128. DOI: https://dx.doi.org/ 10.1080/00461520.1998.9653294

Dobzhansky, T. (1973). Nothing in Biology Makes Sense except in the Light of Evolution. The American Biology Teacher, 35(3), 125-129.

Futuyma, D. J. (2015). Can modern evolutionary theory explain macroevolution? En E. Serrelli, N. Gontier (Eds.), Macroevolution explanation, interpretation and evidence, pp. 29-85. Cham, Suiza. Springer International Publishing.

Gibson, D. J. (1996). Textbook misconceptions: The climax concept of succession. The American Biology Teacher, 58(3), 135-140. DOI: https://dx.doi.org/10.2307/4450101

Gould, S. J. (1980). The evolutionary biology of constraint. Daedalus, 109, 39-52.

González-Galli, L. y Meinardi E. (2017). Obstáculos para el aprendizaje del modelo de evolución por selección natural en estudiantes universitarios de biología. Revista Eureka sobre Enseñanza y Divulgación de las Ciencias, 14(2), 435-449.

Gregory, T. R. (2009). Understanding natural selection: essential concepts and common misconceptions. Evolution: Education and Outreach, 2(2), 156-175. DOI: https:// dx.doi.org/10.1007/s12052-009-0128-1

Hernández, M. C., Álvarez-Pérez, E. y Ruiz-Gutiérrez, R. (2009). La selección natural: aprendizaje de un paradigma. Teorema: Revista Internacional de Filosofía, 2, 107121.

Hey, J. (1999). The neutralist, the fly and the selectionist. Trends in Ecology \& Evolution, 14(1), 35-38. DOI: https://dx.doi.org/10.1016/S0169-5347(98)01497-9

Hiatt, A., Davis, G. K., Trujillo, C., Terry, M., French, D. P., Price, R. M. y Perez, K. E. (2013). Getting to evo-devo: concepts and challenges for students learning evolutionary developmental biology. CBE-Life Sciences Education, 12(3), 494-508. DOI: https:// dx.doi.org/10.1187/cbe.12-11-0203

Ibáñez, M. T. y Martínez-Aznar, M. M. (2005). Solving problems in genetics (II): Conceptual restructuring. International Journal of Science Education, 27(12), 1495-1519. DOI: https://dx.doi.org/10.1080/09500690500186584

Jablonka, E. y Lamb, M. J. (2010). Transgenerational epigenetic inheritance. Evolution: The extended synthesis, 137-174. DOI: https://dx.doi.org/9780262513678.003.0007 
Jeffery, K. R. y Roach. L. E. (1994). A study of the presence of evolutionary protoconcepts in pre-high school textbooks. Journal of Research in Science Teaching, 31(5), 507-518. DOI: https://dx.doi.org/10.1002/tea.3660310506

Jiménez-Aleixandre, M. P. (1994). Teaching evolution and natural selection: a look at textbooks and teachers. Journal of Research in Science Teaching, 31(5), 519-535. DOI: https://dx.doi.org/10.1002/tea.3660310507

King, C. J. H. (2010). An analysis of misconceptions in science textbooks: Earth science in England and Wales. International Journal of Science Education, 32(5), 565-601. DOI: https://dx.doi.org/10.1080/09500690902721681

Linhart, Y. B. (1997). The teaching of evolution-we need to do better. BioScience, 47(6), 385-391. DOI: https://dx.doi.org/10.2307/1313153

Linnenbrink, E. A. y Pintrich, P. R. (2002). Motivation as an enabler for academic success. School Psychology Review, 31(3), 313-327.

Martínez Bonafé J. (2002). Políticas del libro escolar. Madrid. Ediciones Morata.

Martínez Losada, C. y García Barros, S. (2003). Las actividades de primaria y ESO incluidas en libros escolares. Enseñanza de las Ciencias, 21(2), 243-264.

Maskiewicz A. C., Lineback J. E. (2013) Misconceptions are "so yesterday!". CBE-Life Sciences Education, 12(3), 352-356. DOI: https://dx.doi.org/10.1187/cbe.13-01-0014

Mayr, E. (1982). The growth of biological thought: Diversity, evolution, and inheritance. Harvard University Press.

Müller, G. B. (2007). Evo-devo: extending the evolutionary synthesis. Nature Reviews Genetics, 8(12), 943-949. DOI: https://dx.doi.org/10.1038/nrg2219

Munson, B. H. (1994). Ecological misconceptions. The Journal of Environmental Education, 25(4), 30-34.

National Research Council. NRC (1996) National science education standards. National Academy of Sciences.

Nehm, R. H. y Reilly, L. (2007). Biology majors' knowledge and misconceptions of natural selection. BioScience, 57(3), 263-272. DOI: https://dx.doi.org/10.1641/B570311

Occelli, M. y Valeiras, N. (2013). Los libros de texto de ciencias como objeto de investigación: una revisión bibliográfica. Enseñanza de las Ciencias, 31(2), 133-152. DOI: https:// dx.doi.org/10.5565/rev/ec/v31n2.761

Pardo Santano, P. (2004). ¿Qué actividades proponen los libros de texto elaborados para enseñar Geología? Pulso, 27, 49-60.

Perales, F. J. y Jiménez, J. D. (2002). Las ilustraciones en la enseñanza-aprendizaje de las ciencias. Análisis de libros de texto. Enseñanza de las Ciencias, 20(3), 369-386.

Pigliucci, M. y Finkelman, L. (2014). The extended (evolutionary) synthesis debate: where science meets philosophy. BioScience, 64(6), 511-516. DOI: https://dx.doi. org/10.1111/j.1558-5646.2007.00246.x

Pintó, R., Aliberas i Maymí, J. y Gómez Carrillo, R. (1996). Tres enfoques de la investigación sobre concepciones alternativas. Enseñanza de las Ciencias, 14(2), 221-232.

Posner, G. J., Strike, K. A., Hewson, P. W. y Gertzog, W. A. (1982). Accommodation of a scientific conception: Toward a theory of conceptual change. Science education, 66(2), 211-227. DOI: https://dx.doi.org/10.1002/sce.3730660207 
Rees, P. A. A. (2007). The evolution of textbook misconceptions about Darwin. Journal of Biological Education, 41(2), 53-55. DOI: https://dx.doi.org/10.1080/00219266.200 7.9656062

San Román, S. (2013). Evolución de los modelos metodológicos y su relación con la política educativa en España. Educação e Pesquisa, 39(1). DOI: https://dx.doi.org/10.1590/ S1517-97022013000100015

Shtulman, A. (2006). Qualitative differences between naïve and scientific theories of evolution. Cognitive Psychology, 52(2), 170-194. DOI: https://dx.doi.org/10.1016/j. cogpsych.2005.10.001

Smith III, J. P., Disessa, A. A. y Roschelle, J. (1994). Misconceptions reconceived: A constructivist analysis of knowledge in transition. The Journal of the Learning Sciences, 3(2), 115-163. DOI: https://dx.doi.org/10.1207/s15327809jls0302_1

Solbes, J. (2009). Dificultades de aprendizaje y cambio conceptual, procedimental y axiológico (I): Resumen del camino avanzado. Revista Eureka sobre Enseñanza y Divulgación de las Ciencias, 6(1), 2-20.

Stern, L. (2004). Effective assessment: Probing students' understanding of natural selection. Journal of Biological Education, 39(1), 12-17. DOI: https://dx.doi.org/10.1080/002 19266.2004.9655948

Tekkaya, C. (2002). Misconceptions as barrier to understanding biology. Hacettepe Üniversitesi Eğitim Fakültesi Dergisi, 23, 259-266.

Tshuma, T. y Sanders, M. (2015). Textbooks as a possible influence on unscientific ideas about evolution. Journal of Biological Education, 49(4), 354-369. DOI: https:// dx.doi.org/10.1080/00219266.2014.967274

Van Den Broek, P. y Kendeou, P. (2008). Cognitive processes in comprehension of science texts: the role of co-activation in confronting misconceptions. Applied Cognitive Psychology, 22(3), 335-351. DOI: https://dx.doi.org/10.1002/acp.1418

Viennot, L. (1979). Spontaneous reasoning in elementary dynamics. European Journal of Science Education, 1(2), 205-221. DOI: https://dx.doi. org/10.1080/0140528790010209

Woodward, A., Elliott, D. L. (1987). Evolution and creationism in high school textbooks. The American Biology Teacher, 49(3), 164-170. DOI: https://dx.doi.org/10.2307/4448468 


\section{Material anexo}

Tabla ANEXA 1: Listado de libros de texto analizados clasificados por etapa legislativa, con año de publicación, curso y asignatura en la que se utilizaban, editorial y autor. Son un total de 32 libros, 8 para cada etapa legislativa

\begin{tabular}{|c|c|c|c|c|}
\hline Curso & Editorial & Asignatura & Año & Autor \\
\hline \multicolumn{5}{|c|}{ Ley MOYANO } \\
\hline $10 \mathrm{BAC}$ & LUIS VIVES & Ciencias cosmológicas & 1945 & - \\
\hline 3윽 & LUIS VIVES & Ciencias cosmológicas & 1947 & - \\
\hline $10 \mathrm{BAC}$ & PUEYO & Ciencias naturales & 1955 & - \\
\hline $2 \circ \mathrm{BAC}$ & PUEYO & Ciencias naturales & 1957 & - \\
\hline $50 \mathrm{BAC}$ & EDELVIVES & Ciencias naturales & 1960 & - \\
\hline $10 \mathrm{BAC}$ & VICENS VIVES & Ciencias naturales & 1967 & Virgili y Fernández-Polo \\
\hline $50 \mathrm{BAC}$ & MARFIL & Ciencias naturales & 1967 & Esteve \\
\hline $5 \circ \mathrm{BAC}$ & SM & Ciencias Naturales & 1973 & Lerguburu y Barrutia \\
\hline \multicolumn{5}{|c|}{ Ley GENERAL } \\
\hline $\mathrm{COU}$ & ECIR & Biología & 1975 & Dualde \\
\hline $\mathrm{COU}$ & ANAYA & Biología & 1978 & Fernández \\
\hline 8 을 & NATURALIA & Ciencias naturales & 1987 & Abervuj \\
\hline $\mathrm{COU}$ & ANAYA & Biología & 1989 & Mensua, Berges, Carrión, Gil y Martínez \\
\hline $10 \mathrm{BACH}$ & ANAYA & Ciencias naturales & 1989 & Barutell, Berges, Carrión y Martínez \\
\hline $10 \mathrm{BUP}$ & VICENS VIVES & Ciencias naturales & 1990 & $\begin{array}{l}\text { Fernández, López-Puigcerver, Mingo, } \\
\text { Rodríguez, de la Rubia, Torres }\end{array}$ \\
\hline $10 \mathrm{BUP}$ & EVEREST & Ciencias Naturales & 1992 & Miguel, del Cañizo y Costa \\
\hline $10 \mathrm{BUP}$ & $\mathrm{ECIR}$ & Ciencias naturales & 1992 & García, García, Furió, Millán y Sendra \\
\hline \multicolumn{5}{|l|}{ LOGSE } \\
\hline 4 으.S.O. & ANAYA & Biología y geología & 1998 & Berges, Carrión y Gil \\
\hline $10 \mathrm{BACH}$ & EVEREST & Biología y geología & 1998 & Miguel, del Cañizo, Pérez y García \\
\hline $10 \mathrm{BACH}$ & ANAYA & Biología y geología & 2000 & Pulido, Roiz y Rubio \\
\hline $10 \mathrm{BACH}$ & ECIR & Biología y geología & 2002 & García, Carrillo, Furió, García y Sendra \\
\hline $10 \mathrm{BACH}$ & SANTILLANA & Biología y geología & 2002 & Jimeno, Brusi y Ballesteros \\
\hline 4 으.S.S.O. & VICENS VIVES & Biología y geología & 2003 & Fernández, Mingo, Rodríguez y Torres \\
\hline 4을.S.O. & BRUÑO & Biología y geología & 2003 & $\begin{array}{l}\text { Panadero, Lozano, Argüello, Olazábal, } \\
\text { Pérez y Fuente }\end{array}$ \\
\hline 4 으.S.O. & MCGRAWHILL & Biología y geología & 2004 & Calvo y Albarracín \\
\hline \multicolumn{5}{|c|}{ LOE/LOMCE } \\
\hline 4 으.S.O. & SANTILLANA & Biología y geología & 2008 & $\begin{array}{l}\text { Madrid, Meléndez, Blanco y Vidal- } \\
\text { Abarca }\end{array}$ \\
\hline $10 \mathrm{BACH}$ & BRUÑO & Biología y geología & 2015 & $\begin{array}{l}\text { Panadero, Lozano, Olazábal, Argüello, } \\
\text { Argüello y Fuente }\end{array}$ \\
\hline $10 \mathrm{BACH}$ & ANAYA & Biología y geología & 2015 & $\begin{array}{l}\text { Plaza, Gómez, Martínez, Medina, } \\
\text { Martínez-Aedo y Casamayor }\end{array}$ \\
\hline $10 \mathrm{BACH}$ & SANTILLANA & Biología y geología & 2015 & Castillo, Meléndez y Madrid \\
\hline 4으. E.S.O. & VICENSVIVES & Biología y geología & 2016 & $\begin{array}{l}\text { Torres, Argüello, Celiz, Mingo, Olazábal, } \\
\text { Rodríguez y Santos }\end{array}$ \\
\hline
\end{tabular}


Tabla ANEXA 1: Continuación

\begin{tabular}{lllll}
\hline Curso & Editorial & Asignatura & Año & \multicolumn{1}{c}{ Autor } \\
\hline 4o E.S.O. & MCGRAWHILL & Biología y geología & 2016 & Ramos, Colodrón, Serrano y Tomé \\
40 E.S.O. & SANTILLANA & Biología y geología & 2016 & Carrillo y Madrid \\
10 BACH & VICENS VIVES & Biología y geología & 2016 & Torres, Mingo, Olazábal y Santos \\
\hline
\end{tabular}

Tabla ANEXA 2: Teorías, hipótesis y tendencias del pensamiento evolutivo que han intentado explicar la evolución y su aparición en la secuencia de libros analizada. La " $x$ " muestra en qué libros según el año de publicación se han citado las diferentes teorías

\begin{tabular}{|c|c|c|c|c|c|c|c|c|c|c|c|c|c|c|c|c|c|c|c|c|c|}
\hline & Moyano & \multicolumn{6}{|c|}{ General } & & \multicolumn{6}{|c|}{ LOGSE } & \multicolumn{7}{|c|}{ LOE/LOMCE } \\
\hline & \multicolumn{21}{|c|}{ 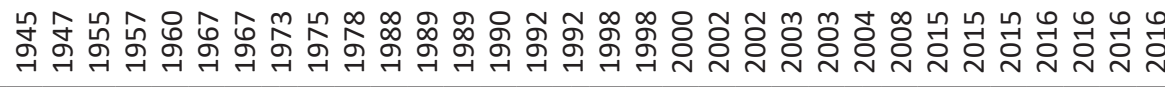 } \\
\hline Fijismo & & & $\mathbf{x}$ & & $x \times$ & $x \times$ & $\mathrm{x}$ & $\mathbf{x}$ & $\mathrm{x}$ & & & $\mathbf{x}$ & $\mathbf{x}$ & $\mathbf{x}$ & $\mathrm{x}$ & $x \times$ & $x$ & $\mathrm{x}$ & $\mathrm{x}$ & $\mathbf{x}$ & \\
\hline Catastrofismo & & & $\mathbf{x}$ & $\mathbf{x}$ & $x$ & $x \times$ & & $\mathbf{x}$ & $\mathbf{x}$ & $\mathbf{x}$ & $\mathbf{x}$ & $\mathbf{x}$ & $\mathbf{x}$ & $\mathbf{x}$ & $\mathbf{x}$ & $x \quad x$ & & $\mathbf{x}$ & & $\mathbf{x}$ & $\mathbf{x}$ \\
\hline Lamarckismo & & $\mathbf{x}$ & $\mathbf{x}$ & $\mathbf{x}$ & $x \quad x$ & $x \times$ & $\mathbf{x}$ & $\mathbf{x}$ & $\mathbf{x}$ & $\mathbf{x}$ & $\mathbf{x}$ & $\mathbf{x}$ & $\mathbf{x}$ & $\mathbf{x}$ & $\mathbf{x}$ & $x \quad x$ & $\mathbf{x}$ & $\mathbf{x}$ & $\mathbf{x}$ & $\mathbf{x}$ & \\
\hline Darwinismo & & $\mathbf{x}$ & $\mathbf{x}$ & $\mathbf{x}$ & $x \quad x$ & $x \times$ & $\mathbf{x}$ & $\mathbf{x}$ & $\mathbf{x}$ & $\mathbf{x}$ & $\mathbf{x}$ & $x \quad x$ & $\mathbf{x}$ & $\mathbf{x}$ & $\mathbf{x}$ & $x \quad x$ & $x \quad x$ & $\mathbf{x}$ & $\mathbf{x}$ & $\mathbf{x}$ & $\mathbf{x}$ \\
\hline Mutacionismo & & & & & & & & & & & & & $\mathbf{x}$ & & & & & & & & \\
\hline Teoria sintética & & $\mathbf{x}$ & $\mathbf{x}$ & $\mathbf{x}$ & $x \times$ & $x \times$ & $x$ & $\mathbf{x}$ & $\mathbf{x}$ & $\mathbf{x}$ & $\mathbf{x}$ & $x \quad x$ & $\mathbf{x}$ & $\mathbf{x}$ & $\mathbf{x}$ & $x \quad x$ & $\mathbf{x}$ & $\mathbf{x}$ & $\mathbf{x}$ & $\mathbf{x}$ & $x$ \\
\hline Neolamarckismo & & & & & & & & & $\mathbf{x}$ & & & $\mathrm{x}$ & & & & & & & & & \\
\hline Neutralismo & & & & & $\mathbf{x}$ & & & & & & $\mathbf{x}$ & $\mathbf{x}$ & $\mathbf{x}$ & & & & $\mathbf{x}$ & $\mathbf{x}$ & $\mathbf{x}$ & $\mathbf{x}$ & \\
\hline Eq. puntuado & & & & & $\mathbf{x}$ & & & & & $\mathbf{x}$ & $\mathbf{x}$ & $\mathbf{x}$ & $\mathbf{x}$ & & $\mathbf{x}$ & $\mathbf{x}$ & $\mathbf{x}$ & $\mathbf{x}$ & $\mathbf{x}$ & $\mathbf{x}$ & $\mathbf{x}$ \\
\hline Finalismo & & & & & & & & & & & $\mathbf{x}$ & & & & & & & & & & \\
\hline Sociobiologia & & & & & & & & & & & $\mathbf{x}$ & $\mathbf{x}$ & & & & & & & & & \\
\hline Evo-devo & & & & & & & & & & & & & & & & & & & & & $\mathbf{x}$ \\
\hline Eco-evo-devo & & & & & & & & & & & & & & & & & & & & & \\
\hline Síntesis extendida & & & & & & & & & & & & & & & & & & & & & \\
\hline
\end{tabular}

Tabla ANEXA 3: Listado de todas las citas a autores encontradas en los libros analizados. La " $x$ " muestra en qué libros según el año de publicación se han citado los diferentes autores

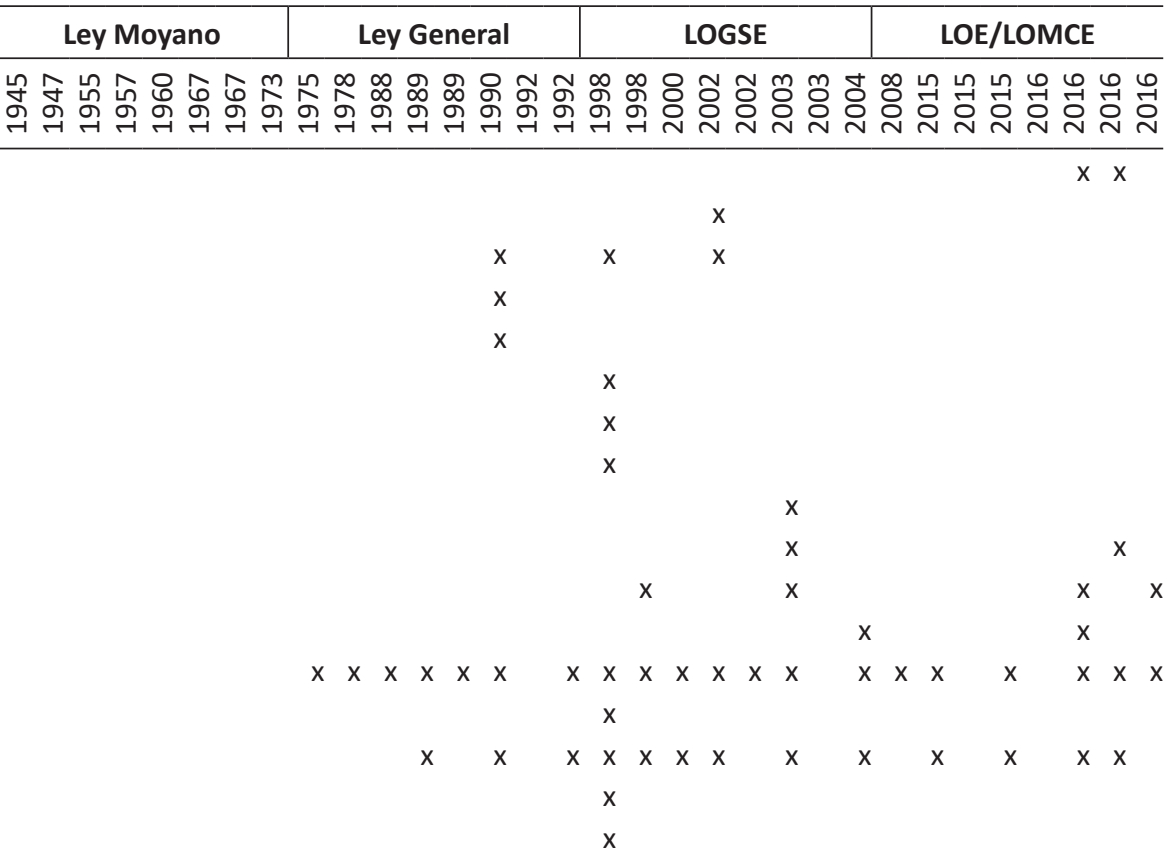


Tabla ANEXA 3: Continuación

\begin{tabular}{|c|c|c|c|}
\hline Ley Moyano & Ley General & LOGSE & LOE/LOMCE \\
\hline
\end{tabular}

\begin{tabular}{|c|c|c|c|c|c|c|c|c|c|c|c|c|c|c|c|c|c|c|c|c|c|c|}
\hline Bentham & & & & & & & & & & $x$ & & $x$ & & & & & & & & & & \\
\hline Félix de Ázara & & & $x$ & & & & & & & & & & & & & & & & & & & \\
\hline Lamarck & & $x$ & $x$ & $x$ & $x$ & $x$ & $x$ & $x$ & $x$ & $x \quad x$ & $x \quad x$ & & & $x$ & $\mathrm{x}$ & $x$ & $x>$ & $x$ & $\mathrm{x}$ & $x$ & $x$ & $x$ \\
\hline Malthus & & & & & $x$ & $x$ & & & & $x \quad x$ & $x$ & & & $x$ & & $x$ & & $x$ & & & $x$ & \\
\hline Darwin & & $\mathrm{x}$ & $x$ & $x$ & $x$ & $x$ & $x$ & $x$ & $x$ & $x \quad x$ & $x \quad x$ & $x$ & $x$ & $x$ & $\mathrm{x}$ & $x$ & $x>$ & $x$ & $x$ & $x \quad x$ & $x$ & $x$ \\
\hline Wallace & & & $x$ & $x$ & $x$ & $x$ & $x$ & & $x$ & $x$ & $x$ & $x$ & & $x$ & & $x$, & $x>$ & $x$ & & $x$ & $x$ & $x$ \\
\hline Lyell & & & & & $x$ & & & & & $x \quad x$ & $x$ & & & $x$ & & $x$ & & $x$ & & $x$ & & \\
\hline Huxley & & & & & & & & & & $x$ & & $x$ & & & & & & & & & & \\
\hline Hooker & & & & & & & & & & $x$ & & & & & & & & & & & & \\
\hline Kelvin & & & & & & & & & & & & $x$ & & & & & & & & & & \\
\hline Haeckel & & $x$ & & & $x$ & $x$ & & & $x$ & $x$ & $x$ & $x$ & & $x$ & & & & & & & & \\
\hline Owen & & $x$ & & & & & & & & & & & & & & & & & & & & \\
\hline Mendel & $x$ & $x$ & & $x$ & $x$ & & & $x$ & & $x$ & $x$ & & & $x$ & & & $x$ & & & $x$ & & \\
\hline Weismann & $x$ & & & & & & & & & & & $x$ & & & & & & & & & & \\
\hline Garstand & & & & & & & & & & & & $x$ & & & & & & & & & & \\
\hline Hardy & & $x$ & & & $x$ & & & & & $x$ & & & & $x$ & & & & & & & & \\
\hline Weinberg & & $\mathrm{x}$ & & & $x$ & & & & & $x$ & & & & $x$ & & & & & & & & \\
\hline Bateson & & & & & & & & & & & & & & $x$ & & & & & & & & \\
\hline Corrend & & & & & & & & & & & & & & $x$ & & & & & & & & \\
\hline de Vries & & $x$ & & & & & & & & $x$ & $x$ & $x$ & & $x$ & & & & & & & & \\
\hline Morgan & & $x$ & & & & & & & & & & $x$ & & & & & & & & & & \\
\hline Fisher & & & & & $x$ & & & & & $x$ & & & & $x$ & & & & & & & & \\
\hline Wrigth & & $x$ & & & $x$ & & & & & $x$ & & & & $x$ & & & & & & & & \\
\hline Haldane & & & & & $x$ & & & & & $x$ & & & & $x$ & & & & & & & & \\
\hline Chetverikov & & & & & $x$ & & & & & & & & & & & & & & & & & \\
\hline Dobzhansky & & & & & $x$ & & & & & $x$ & $x$ & $x$ & $x$ & $x$ & & & & & & $x$ & $x$ & $x$ \\
\hline Mayr & & & & & & & & & & $x$ & & $x$ & $x$ & $x$ & & & & & & & $x$ & $x$ \\
\hline Huxley & & & & & & & & & & $x$ & & & & $x$ & & & & & & & $x$ & $x$ \\
\hline Simpson & & & & & & & & & & $x$ & & $x$ & $x$ & $x$ & & & & & & & & \\
\hline Kettlewell & & & & & & & $x$ & & & & & $x$ & & & $x$ & & & & & & & \\
\hline Le gros clark & & & & & & & & & & & & $x$ & & & & & & & & & & \\
\hline Weiner & & & & & & & & & & & & $x$ & & & & & & & & & & \\
\hline Whittaker & & & & & & & $x$ & & & $x$ & & & & & & & & & & & & \\
\hline Kimura & & & & & $x$ & & & & & & & $x$ & & $x$ & & & & & $x$ & & & $x$ \\
\hline Eldrege & & & & & & & & & & & $x$ & $x$ & & $x$ & & & & $x$ & & & & $x$ \\
\hline Gould & & & & & $x$ & & & & & & $x$ & $x$ & & $x$ & & $x$ & & $x$ & $x$ & & & $x$ \\
\hline Goldschmidt & & & & & $x$ & & & & & & & & & & & & & & & & & \\
\hline Grassé & & & & & & & & & & & $x$ & & & & & & & & & & & \\
\hline Dawkins & & & & & & & & & & & $x$ & & & & & & & & & & & \\
\hline Stanley & & & & & & & & & & & & & & & & & & $x$ & & & & \\
\hline Lewontin & & & & & & & & & & & & $x$ & & & & & & & & & & \\
\hline Alvárez & & & & & & & & & & $x$ & & & & & & & & & & & & \\
\hline Ansaro & & & & & & & & & & $x$ & & & & & & & & & & & & \\
\hline Ostrom & & & & & & & & & & $x$ & & & & & & & & & & & & \\
\hline Stainer & & & & & & & & & & $x$ & & & & & & & & & & & & \\
\hline Margulis & & & & & & & & & & $x$ & & $x$ & & & & & & & & $x$ & & $x$ \\
\hline Ayala & & & & & & & & & & & & $x$ & & & & & & & & & & \\
\hline Wintrebert & & & & & & & & & & $x$ & & & & & & & & & & & & \\
\hline Grassé & & & & & & & & & & $x$ & & & & & & & & & & & & \\
\hline Bourdier & & & & & & & & & & $x$ & & & & & & & & & & & & \\
\hline
\end{tabular}


Tabla ANEXA 4: Aparición en los libros de texto de algunas figuras explicativas clásicas del tema de evolución. Este tipo de figuras en forma de esquema aclarativo o ejemplo práctico se clasificaron como figuras con contenido aclarativo, complementario o de ampliación. La " $x$ " muestra en qué libros según el año de publicación aparecen

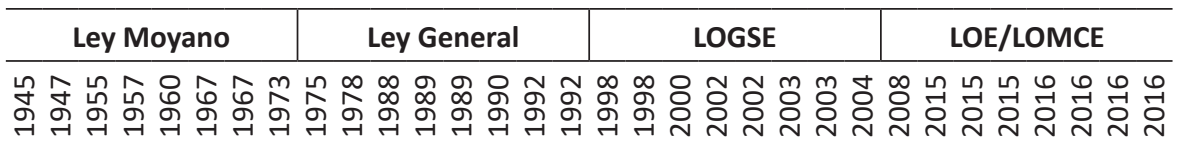

Polilla del

abedul

Picos Pinzones

Diagrama

Marsh

Cuello Jirafas

Ontogenia

Analogías

Homologías

Archaeopteryx

Reloj de la

Tierra

Trompa Ele-

fantes

$\begin{array}{llllllllll}x & x & x & x & x & x\end{array}$

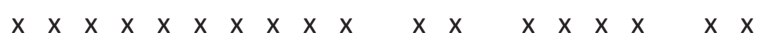

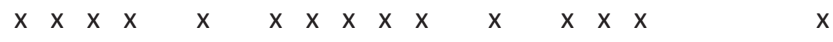

$x \quad x$

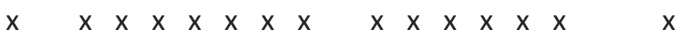

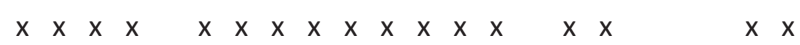

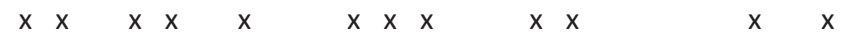

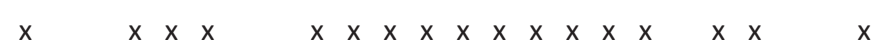

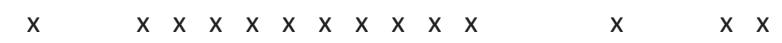

$\begin{array}{llllll}x & x & x & x & x\end{array}$

$\mathrm{X}$

$\mathrm{X}$ 